\title{
RECONSTRUCTION OF RAT CALVARIAL DEFECTS WITH HUMAN MESENCHYMAL STEM CELLS AND OSTEOBLAST-LIKE CELLS IN POLY-LACTIC-CO-GLYCOLIC ACID SCAFFOLDS
}

\author{
Chen Zong ${ }^{1}$, Deting Xue ${ }^{2}$, Wenji Yuan ${ }^{1}$, Wei Wang ${ }^{3}$, Dan Shen ${ }^{4}$, Xiangmin Tong ${ }^{4}$, Dongyan Shi ${ }^{1}$, Liyue Liu ${ }^{1}$, Qiang \\ Zheng ${ }^{2}$, Changyou $\mathrm{Gao}^{3}$, and Jinfu Wang ${ }^{1 *}$ \\ ${ }^{1}$ Laboratory of Stem Cells, Institute of Cell Biology, College of Life Sciences, Zhejiang University, Hangzhou, \\ Zhejiang 310058, P. R. China \\ ${ }^{2}$ Institute of Orthopedics, The Second Hospital, Zhejiang University, Hangzhou, Zhejiang 310009, P. R. China. \\ ${ }^{3}$ Institute of Medical Materials, College of Material and Chemistry, Zhejiang University, Hangzhou, Zhejiang \\ 310028, P. R. China \\ ${ }^{4}$ Laboratory of Bone Marrow, The First Hospital, Zhejiang University, Hangzhou, Zhejiang 310006, P. R. China
}

\begin{abstract}
Human mesenchymal stem cells (hMSCs) can be used for xenogenic transplantation due to their low immunogenicity, high proliferation rate, and multi-differentiation potentials. Therefore, hMSCs are an ideal seeding source for tissue engineering. The present study evaluates the reconstruction effects of hMSCs and osteoblast-like cells differentiated from hMSCs in poly-lactic-co-glycolic acid (PLGA) scaffolds on the calvarial defect of rats. Two bilateral fullthickness defects $(5 \mathrm{~mm}$ in diameter) were created in the calvarium of nonimmunosuppressed Sprague-Dawley rats. The defects were filled by PLGA scaffolds with hMSCs (hMSC Construct) or with osteoblast-like cells differentiated from hMSCs (Osteoblast Construct). The defects without any graft (Blank Defect) or filled with PLGA scaffold without any cells (Blank Scaffold) were used as controls. Evaluation was performed using macroscopic view, histology and immunohistochemical analysis respectively at 10 and 20 weeks after transplantation. In addition, fluorescent carbocyanine CMDil was used to track the implanted cells in vivo during transplantation. The results showed that while both hMSC Construct and Osteoblast Construct led to an effective reconstruction of critical-size calvarial defects, the bone reconstruction potential of hMSC Construct was superior to that of Osteoblast Construct in non-autogenous applications. Our findings verify the feasibility of the use of xenogenic MSCs for tissue engineering and demonstrate that undifferentiated hMSCs are more suitable for bone reconstruction in xenotransplantation models.
\end{abstract}

Keywords: Human mesenchymal stem cells, tissue engineering, xenotransplantation, bone reconstruction.

\footnotetext{
* Address for correspondence:

Jinfu Wang

Laboratory of Stem Cells, Institute of Cell Biology,

College of Life Sciences, Zhejiang University

388, Yuhangtang Road, Hangzhou, Zhejiang 310058

P. R. China
}

E-mail address: wjfu@zju.edu.cn

\section{Introduction}

There is a significant need for therapies to enhance healing in large bone defects caused by trauma, congenital deformity and tumor resection because each year over 1 million cases of patients need bone graft procedures to correct such defects in the USA(Langer and Vacanti, 1993; Holtorf et al., 2006). Three primary approaches for bone defect repair are autograft bone, allograft bone and tissue engineered bone transplantations (Kubler et al., 1995). However, transplantation of autograft bone or allograft bone is not optimal due to the limitation of supply and the risk of morbidity (Younger and Chapman, 1989; Hao et al., 2010). Bone tissue engineering, as a practical and promising method of bone defect regeneration, has become of major interest for bone reconstruction to repair large bone defects.

Mesenchymal stem cells (MSCs) derived from bone marrow are an obvious source of autologous stem cells, and used as seeding cells for cell therapy and tissue engineering (Bruder et al., 1998a; Bruder et al., 1998b; Kon et al., 2000; Cui et al., 2007). MSCs have a strong regeneration potential and immunosuppressive properties that are important for allografts. In fact, MSCs have already been employed for clinical trials in a number of contexts, such as the facilitation of hematopoietic and immune reconstitution after hematopoietic stem cell transplantation (Koc et al., 2000; Lazarus et al., 2005), construction of the vessel wall (Gong and Niklason, 2008), as well as the regeneration of cartilage using tissue engineering techniques (Jorgensen et al., 2004; Bernardo et al., 2007). Furthermore, a number of studies have demonstrated that autologous MSCs cultured in scaffolds can induce new bone formation in vivo and lead to improved healing of critical-size defects (Yamada et al., 2004; Meinel et al., 2005; Mankani et al., 2006; Miura et al., 2006). In these studies, MSCs are always induced into osteoblast-like cells with the osteogenic medium before transplantation. Osteoblast-like cells derived from MSCs share similar characters with osteoblasts (Kassem et al., 1991). Osteoblast-like cells have a good ability of growth and proliferation and keep their biological characteristics constant after several passages. In addition, osteoblastlike cells can secrete much bone matrix to form mature bone with deposition of calcium salts, and play an important role in osteogenesis and bone regeneration. 
However, osteoblast-like cells lose some characteristics and multipotential of differentiation as MSCs. Recent studies also verify the ability of undifferentiated MSCs in scaffolds to enhance the bone formation and repair defects of bone tissues in vivo (Korda et al., 2008; Niemeyer et al., 2010a). Therefore, it is interesting to compare the ability of bone reconstruction between these two cell types.

In this study, we used porous poly-lactic-co-glycolic acid (PLGA) scaffolds as carriers. PLGA is a biodegradable porous material used commonly in tissue engineering. It can be removed from the body by normal metabolic pathways and is relatively harmless to the cells and tissues (Gopferich, 1996). We seeded hMSCs into scaffolds, and then expanded and induced osteogenically hMSCs in scaffolds. Finally, the bone reconstruction ability of undifferentiated hMSCs and osteoblast-like cells differentiated from hMSCs in scaffolds was comparatively analyzed through the reconstruction experiment of calvarial defects in nonimmunosuppressed SpragueDawley (SD) rats.

\section{Materials and Methods}

\section{Preparation of experimental materials}

Porous poly-lactic-co-glycolic acid (PLGA) was fabricated into porous scaffolds by a porogen-leaching technique with gelatin particles as the porogen (Zhou et al., 2005). Briefly, the notable feature of this process was that the gelatin particles bound together to form a three-dimensional assembly through a water vapor treatment before the polymer solution was cast. The PLGA scaffolds were prepared as cylinders $(10 \times 3 \mathrm{~mm})$ and have a porosity of $85 \%$. The pore diameter was about $280-450 \mu \mathrm{m}$.

Human mesenchymal stem cells (hMSCs) were isolated from the whole bone marrow of three healthy donors at the First Affiliate Hospital, Zhejiang University, using the method described previously (Xie et al., 2005). All donors had given informed consent before collection of their bone marrow. hMSCs were cultured with minimal essential medium $\alpha$ ( $\alpha$-MEM; HyClone, Shanghai, China) supplemented with $10 \%$ fetal bovine serum (FBS; Gibco BRL, Hangzhou, China), 10,000 U/mL penicillin and 10,000 U/mL streptomycin (Life Technologies, Beijing, China). hMSCs were incubated at $37^{\circ} \mathrm{C}$ in a high humidity environment containing $5 \% \mathrm{CO}_{2}$. The medium was replaced every 3 days, and cells were passaged at approximately $80 \%$ confluence. hMSCs in the third passage were prepared for the following experiments.

Human calvarial osteoblasts (HCOs; Yuhengfeng, Beijing, China) were cultured according to the manufacturer's instructions. Briefly, cells were seeded in T-75 flasks at a density of 5,000 cells $/ \mathrm{cm}^{2}$ and cultured with Osteoblast Medium (ObM, Yuhengfeng) composed of $500 \mathrm{~mL}$ basal medium, $25 \mathrm{~mL}$ FBS, $5 \mathrm{~mL}$ osteoblast growth supplement (ObGS) and $5 \mathrm{~mL}$ penicillin/ streptomycin solution $(\mathrm{P} / \mathrm{S})$ at $37^{\circ} \mathrm{C}$ and in a high humidity environment containing $5 \% \mathrm{CO}_{2}$. After 24 hours of culture, unattached cells were discarded by refreshing the medium. Attached HCOs were continued to incubate under above condition. The medium was replaced every 2 days, and cells were passed at about $80 \%$ confluence.

Two-month-old male non-immunosuppressed SD rats with a body weight of 200 to $250 \mathrm{~g}$ were used as transplant recipients for this study. The experimental protocol was approved by the Animal Research Centre at Zhejiang University. Before surgery, the animals were kept in ventilated, clean and standard air conditions at a constant temperature of $22^{\circ} \mathrm{C}$ with a 12-hour light/day cycle. Animals could receive freely drinking water and standard laboratory foods. All animals were cared for according to the policies and principles established by State Scientific and Technological Commission (SSTC).

\section{Cell seeding and culture of Constructs in vitro}

The construct of PLGA-hMSCs (hMSC Construct) was prepared as described previously with a small modification (Yang et al., 2010). Briefly, PLGA scaffolds were sterilized using $70 \%$ ethanol, followed by rinsing the scaffold several times with phosphate buffered saline (PBS). hMSCs in the third passage were suspended in $\alpha$-MEM medium without FBS at a cell density of $2 \times 10^{6}$ cells $/ \mathrm{mL}$. $250 \mu \mathrm{L}$ of hMSC suspension was seeded into the scaffold by a syringe linked with the outlet of a screw-bottom, and then cells were allowed to attach for $2 \mathrm{~h}$ before $3 \mathrm{~mL}$ of $\alpha$-MEM medium with $10 \%$ FBS per well was added into a six-well plate for static culture. After $24 \mathrm{~h}$ of static culture, the constructs were transferred into a perfusion culture system described previously (Yang et al., 2010). The perfusion culture system was incubated at $37^{\circ} \mathrm{C}$ and in a high humidity environment containing $5 \% \mathrm{CO}_{2}$, The flow rate of medium was kept at $0.2 \mathrm{~mL} / \mathrm{min}$. The medium used for perfusion culture was the $\alpha$-MEM medium supplemented with $10 \%$ FBS (growth medium). The medium was pumped from the medium reservoir into the cell-scaffold construct in each culture cassette, and then returned to the medium reservoir through the platinum-cured silicon tubing. Growth medium in the medium reservoir was replaced at a rate of 50\% every 2-3 days. The constructs were measured for DNA content after culture for $0,6,9$, 12 and 15 days, respectively. The optimal culture time was obtained according to the DNA content analysis. Then, this time point was chosen as the preparation time in vitro of hMSC Construct before transplantation.

The construct of PLGA-osteoblast-like cells derived from hMSCs (Osteoblast Construct) was prepared as follows: Firstly, PLGA scaffolds seeded with hMSCs were cultured for 9 days in the perfusion culture system as in the preparation of hMSC Construct. Then, the growth medium was replaced with the osteogenic medium $(\alpha-$ MEM supplemented with $10 \% \mathrm{FBS}, 50 \mu \mathrm{g} / \mathrm{ml}$ ascorbic acid, $10 \mathrm{mM}$ sodium, $\beta$-glycerophosphate, and $10^{-8} \mathrm{M}$ dexamethasone; Sigma, Shanghai, China) for another 14 days of culture. Osteogenic medium was replaced every 4 days. Then, before transplantation, the DNA content of the constructs was measured.

\section{DNA content assay}

The constructs cultured for $0,6,9,12$ and 15 days as well as induced osteogenically for 14 days, were used for DNA 
content assay. Three constructs per time point were removed from the perfusion system and stored in $\mathrm{ddH}_{2} \mathrm{O}$ at $-20^{\circ} \mathrm{C}$ until the assay was performed. For DNA content assay, the frozen scaffolds with cells were thawed at room temperature and homogenized in $1 \mathrm{~mL}$ lysis buffer $(50 \mathrm{mM}$ Tris/HCl, pH7.6, 0.1\% (v/v) Triton X-100). The lysate was assayed for DNA content using a fluorescent dye Hoechst 33258. Briefly, the lysate was sonicated on ice for 30 s and vortexed for 5-10s. After centrifugation, the supernatant was collected. Standards of calf thymus DNA were prepared in the range from 0 to $30 \mu \mathrm{g} / \mathrm{mL}$. TNE buffer (10mM Tris base, $1 \mathrm{mM}$ EDTA and $200 \mathrm{mM} \mathrm{Nacl}$ ) was added to each well of a 96 -well plate at $50 \mu \mathrm{L} /$ well. Then, $50 \mu \mathrm{L}$ standards and samples were added to each well in triplicate. Then, $100 \mu \mathrm{L}$ Hoechst 33258 dye solution $(1 \mu \mathrm{g} /$ $\mathrm{mL}$ ) was added to each well. Finally, the plate was incubated for $10 \mathrm{~min}$ in the dark at room temperature, and then read on Auto Microplate Reader (Infinite M200, Tecan, Austria; Ex350nm/Em450nm). Cell number was determined by correlating DNA with a known amount of cells. Samples were run in triplicate and compared against calf thymus DNA standards.

\section{Assay of osteogenic differentiation in scaffolds}

Alkaline phosphatase (ALP) activity, as an important osteogenic differentiation marker, was detected to verify whether hMSCs in PLGA scaffolds had differentiated to osteoblast-like cells. ALP activity assay was measured using an ALP measurement kit (Jiancheng Biotechnology Institute, Nanjing, China) with disodium phenyl phosphate as a substrate. Briefly, scaffolds with hMSCs undergoing 9-day-culture in the growth medium were continued to culture in perfusion system with the osteogenic medium for another 14 days. Then, constructs $(n=3)$ were moved from the perfusion system and washed three times in PBS, and homogenized in lysis solution $(0.1 \%$ Triton $\mathrm{X}-100$ in PBS) and sonicated for $5 \mathrm{~min}$. After the lysate was centrifuged, $300 \mu \mathrm{l}$ supernatant was added into a $5 \mathrm{ml}$ Corning tube, and then $500 \mu \mathrm{l}$ buffer and $500 \mu \mathrm{l}$ substrate solution were added before incubation at $37^{\circ} \mathrm{C}$ for $15 \mathrm{~min}$. After that, the above mixture was added to $1.5 \mathrm{ml}$ of chromogenic reagent. Finally, the absorbance was measured at $520 \mathrm{~nm}$, and the ALP activity of each sample was calculated using the phenol standard curve.

RT-PCR was performed to determine the expression of specific genes for osteogenesis in scaffolds. As above, the constructs $(n=3)$ were collected and three times washed with PBS. The constructs were pulverized and the total RNA was extracted using Trizol reagent (TaKaRa, Shanghai, China) according to the manufacturer's instructions. RNA $(1 \mu \mathrm{g})$ was used to synthesize first strand cDNA by reverse transcriptase (Fermentas, Shanghai, China). The polymerase chain reactions (PCR) were performed to analyze the genes ALP, collagen I (COL I) and osteopontin (OPN) expressed by human osteoblasts. GAPDH was used as a housekeeper gene to normalize all measurements for the other products. Primers were designed using PRIMER EXPRESS 1.0a Software (Applied Biosystems) as follows: ALP: fwd 5'ACA AGC ACT CCC ACT TCA TC 3', rev 5' ATT CTG CCT CCT
TCC ACC 3'; COL I: fwd 5' TCA AAG GCA ATG CTC AAA CA 3', rev 5' ACA TCAAGA CAA GAA CGA GGT AG 3'; OPN: fwd 5' AGC CGT GGG AAG GAC AGT TAT G3', rev 5' GGA GTT TCC ATG AAG CCA CAA AC 3'; GAPDH: fwd 5' GAA GGT CGG AGT CAA CGG 3', rev 5' GGA AGA TGG TGA TGG GAT T 3'. PCR reaction conditions were $94^{\circ} \mathrm{C}$ for $5 \mathrm{~min}$, then 30 cycles of $94^{\circ} \mathrm{C}$ for $30 \mathrm{~s}, 53^{\circ} \mathrm{C}(\mathrm{ALP}), 43^{\circ} \mathrm{C}(\mathrm{COL} \mathrm{I}), 52^{\circ} \mathrm{C}$ (OPN) or $53^{\circ} \mathrm{C}(\mathrm{GAPDH})$ for $30 \mathrm{~s}$ and then $72^{\circ} \mathrm{C}$ for $1 \mathrm{~min}$, finally followed by $72^{\circ} \mathrm{C}$ for $5 \mathrm{~min}$. PCR products were electrophoresed on a $1.5 \%$ agarose gel and then stained with ethidium bromide. Each PCR product was run three times. Stained bands were visualized under UV light and photographed.

\section{Surgery and transplantation procedure}

All surgery was performed under a protocol approved by SSTC. Round calvarial defects $(5 \mathrm{~mm}$ in diameters, fullthickness) were created in two-month-old SD rats (Center of Experimental Animals, Zhejiang University). After general anesthesia with $4 \%$ chloral hydrate, the skin and underlying tissues of vertex, including the periosteum and the temporalis muscles, were raised to expose the calvaria, and then two full-thickness defects in symmetry to the sagittal suture were generated by a dental bur. Constant saline irrigation was provided and the dura mater was kept intact with minimal invasion during the procedure. The procedure was performed under sterile conditions. After the defects were implanted with grafts, a single layer of soft tissue was closed with absorbable sutures, and then the skin incision was closed with nylon sutures. After surgery, SD rats were kept in the clean conditions with enough water and foods.

$24 \mathrm{SD}$ rats used for the experiment were divided into four groups (see Table 1). The rats were sacrificed by cervical dislocation or euthanasia with an overdose of chloral hydrate, respectively, at 10 and 20 weeks after transplantation, and their calvarias with grafts were harvested.

Table 1. Treatment groups of experimental animals

\begin{tabular}{cccc}
\hline Group & Rat number & $\begin{array}{c}\text { Graft at left } \\
\text { defect }\end{array}$ & $\begin{array}{c}\text { Graft at } \\
\text { right defect }\end{array}$ \\
\hline A & 6 & $\begin{array}{c}\text { hMSC } \\
\text { Construct }\end{array}$ & $\begin{array}{c}\text { Blank } \\
\text { Defect }\end{array}$ \\
B & 6 & $\begin{array}{c}\text { hMSC } \\
\text { Construct }\end{array}$ & $\begin{array}{c}\text { Blank } \\
\text { Scaffold }\end{array}$ \\
C & 6 & $\begin{array}{c}\text { Osteoblast } \\
\text { Construct }\end{array}$ & $\begin{array}{c}\text { Blank } \\
\text { Scaffold }\end{array}$ \\
D & 6 & $\begin{array}{c}\text { Osteoblast } \\
\text { Construct }\end{array}$ & $\begin{array}{c}\text { hMSC } \\
\text { Construct }\end{array}$ \\
\hline
\end{tabular}

hMSC Construct: the scaffold with hMSCs; Osteoblast Construct: the scaffold with osteoblast-like cells differentiated from hMSCs; Blank Defect: the defect without any graft; Blank Scaffold: the scaffold without any cells. 


\section{Cell tracing in vivo}

To in vivo trace hMSCs and osteoblast-like cells in scaffolds grafted into rats, we used the fluorescent carbocyanine CM-Dil to label cell membranes. The method was an improvement on the manufacturer's protocol (Ferrari et al., 2001). Briefly, at 24h before transplantation, hMSCs or osteoblast-like cells in scaffold were labeled with $4 \mu \mathrm{g} / \mathrm{ml} \mathrm{CM-Dil} \mathrm{for} \mathrm{about} 2 \mathrm{~h}$ at $37^{\circ} \mathrm{C}$, and then the scaffold was washed with PBS for three times and continuously cultured in the perfusion culture system until transplantation. Cell tracing was performed at 10 weeks after transplantation. Tissue samples from defect areas were harvested from 4 groups (2 samples per group) and decalcified in Decalcifying Fluid (Zhongshan, Beijing, China) for 4 days at room temperature, and then washed in running water for about 2 hours. The $7 \mu \mathrm{m}$ frozen section of sample was prepared and then counterstained by DAPI for 5 minutes. The result was observed using a Confocal Laser Scanning Microscope (LSM-510, Carl Zeiss, Germany).

\section{Histological evaluation}

Tissue samples harvested from 4 groups (2-4 samples per group) at 10 and 20 weeks after transplantation, respectively, were fixed with $10 \%$ buffered formalin for $24 \mathrm{~h}$ and then several times washed with PBS. The sample was the decalcified in neutral 10\% EDTA solution for half a month at room temperature, and then dehydrated in a graded alcohol series and embedded in paraffin. $5 \mu \mathrm{m}$ sections of the sample were prepared and stained with hematoxylin and eosin (H\&E) and Modified Masson Trichrome as previously described (Garvey, 1984; Cowan et al., 2004). The stained sections were photographed digitally under a microscope (Digital Camera DXM200F, Nikon, Japan). Semi-quantitative image analysis (Ahmad et al., 2004; Zsarnovszky et al., 2005; ) was used to estimate the new forming bone at 20 weeks after transplantation, i.e., to calculate the percentage of newly formed bone within the defect using the computerized image analysis software Image-Pro Plus (IPP) 6.0. Briefly, the image was corrected for the optical density before the region-ofinterest (ROI) was selected in accordance with the color specific for the region of new bone formation in the histological slide. Then the parameters of the ROI, such as area, were calculated. Automation of these steps is included in an algorithm referred as a macro. The macro was used to normalize the selection of other ROIs. The percentage of newly formed bone was determined by ROI area/total area of images. 5 sections from each sample were used for semi-quantitative image analysis. 5 regions in each section were photographed at 200x magnification. A total of 25 images for each sample were digitalized.

Immunohistochemical analysis was performed using the Histostain-Plus IHC Kit (MR Biotech, Shanghai, CHINA) according to the manufacturer's instructions. Briefly, tissue samples from defect areas were harvested from 4 groups (four samples per group) at 20 weeks after transplantation. The thickness of bone-like tissue in the harvested samples was determined by the Micrometer (126-125 TMC-25, Mitutoyo, Japan). Sections of samples were prepared as described above, and then deparaffinized, rehydrated and incubated in 3\% hydrogen peroxide in methanol for $10 \mathrm{~min}$ to inactivate the endogenous peroxidase. After three times washing with PBS, the sections were digested with Trypsin Diluent (Zhongshan, Beijing, China) for 15 min to unmask antigen binding sites and then washed three times with PBS. After that, the sections were incubated with $10 \%$ goat serum for $20 \mathrm{~min}$ to block non-specific binding, followed by incubation with the primary antibody, i.e., rabbit polyclonal antibody against human osteocalcin (OCN, Abcam, Hangzhou, China) overnight at $4^{\circ} \mathrm{C}$. Afterwards, the sections were washed three times with PBS and incubated with biotinylated $\mathrm{IgG}$ against rabbit for $15 \mathrm{~min}$, and then exposed to horseradish peroxidase (HRP) labeled streptavidin for $15 \mathrm{~min}$. After washing three times with PBS, the antibody complex was visualized by addition of a buffered diaminobenzidine (DAB) solution. Then, the sections were counterstained in hematoxylin for $5 \mathrm{~min}$ and mounted with neutral gum. The sections treated only with the second antibody (without primary antibody treatment) were used as a negative control. Finally, the sections were photographed. To evaluate the protein content of human OCN expressed by implanted cells in the graft area, we used IPP as the method of semi-quantitative image analysis on immuno- histochemical section image to measure the Integrated Optical Density (IOD) (Cybernetics, 2002). As above, a total of 25 images per sample were used for calculation.

\section{Statistics}

Experimental results were expressed as means \pm standard deviation (StDev) of the means. All collected data were examined by multifactorial analysis of variance. Differences between the independent variables were checked in post hoc tests (Tukey's studentized range (HSD) tests for variables). All tests were two-tailed and statistical significance was accepted at $P<0.05$.

\section{Results}

\section{Growth of hMSCs in PLGA scaffolds}

The proliferation of cells in the PLGA scaffold was evaluated by determining the double-stranded DNA content. After the static culture for $24 \mathrm{~h}$, the cell density in each PLGA scaffold was detected as $(0.42 \pm 0.08) \times$ $10^{6} \mathrm{cells} / \mathrm{scaffold}$. During perfusion culture, the cell density of hMSCs in PLGA scaffold increased gradually and reached a cell density of $(2.27 \pm 0.27) \times 10^{6}$ cells/scaffold after culturing for 12 days, 5 -fold the initial cell density. Then, the increase in rate decreased after 12 days. There is no significant difference in cell density between day 12 and day $15(P>0.05)$ (Fig. 1A). Therefore, perfusion culture of hMSCs in the scaffold for 12 days in vitro was used to prepare hMSC Construct so as to assure a sufficient cell density and viability for transplantation.

The cell density in PLGA scaffolds after 9 days of perfusion culture with growth medium was $(1.91 \pm 0.17)$ $\times 10^{6} \mathrm{cell} / \mathrm{scaffold}$. After 14 days of culture with osteogenic 

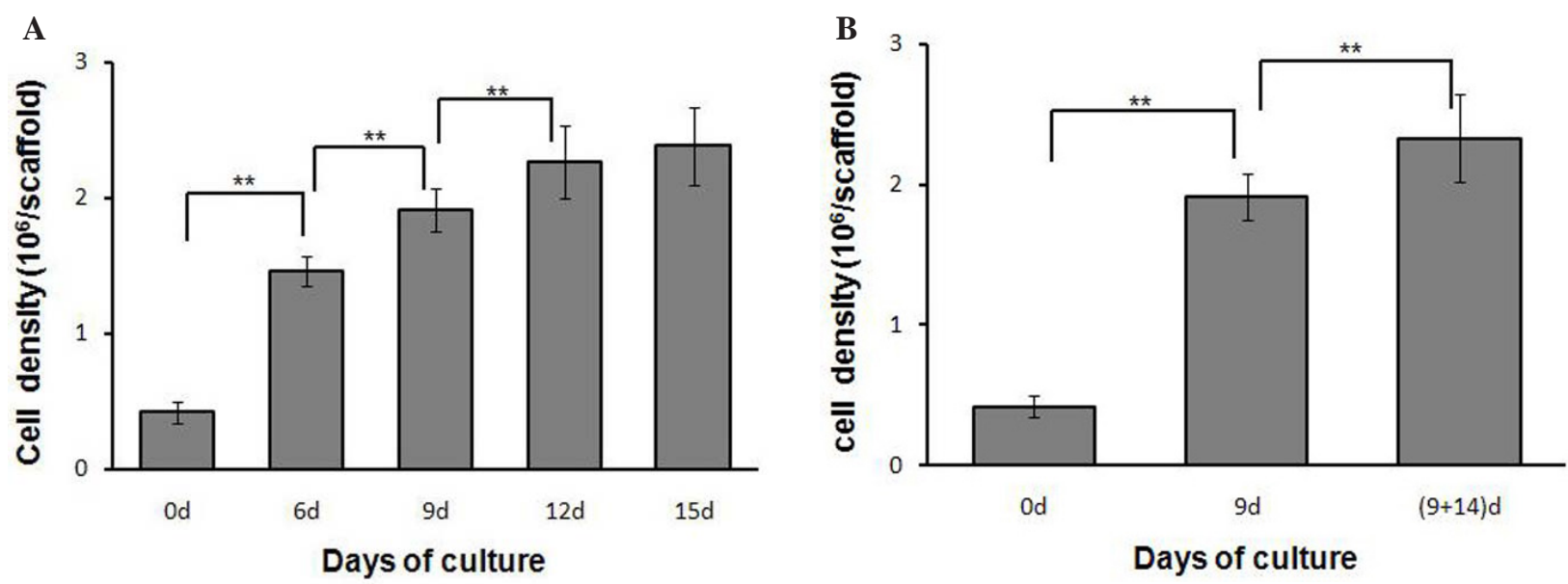

Fig. 1. Proliferation of cells in PLGA scaffold in the perfusion culture system. The cell density in scaffold and the cell proliferation were evaluated by determining the double-stranded DNA (dsDNA) content. (A) Cell densities of hMSCs in PLGA scaffold for culture of 0, 6, 9, 12 and 15 days. (B) Cell densities of hMSCs in PLGA scaffold for culture of 0 and 9 days, and then for induction of 14 days with the osteogenic medium. All the experiments were conducted independently $(n=3)$. Error bars represent SD. The asterisks $(* *)$ indicate statistically significant differences between the relative culture times $(P<0.01)$.

A

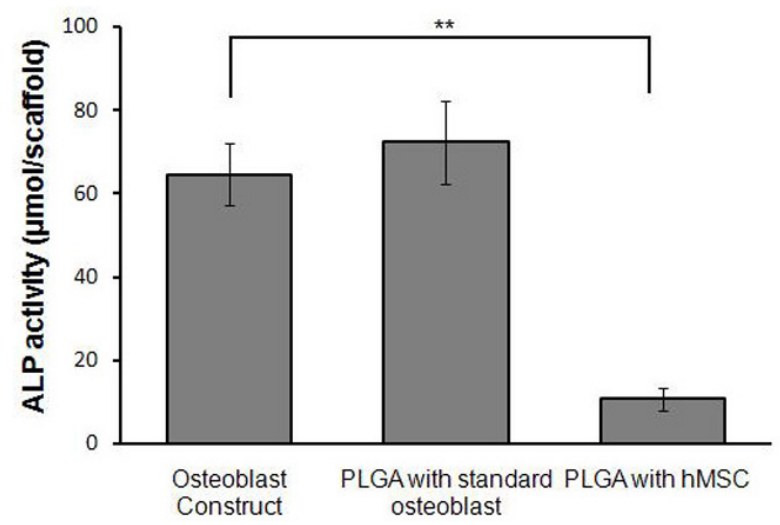

B

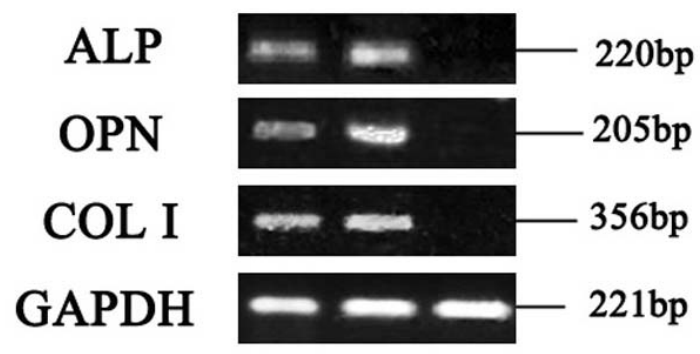

Fig. 2. Osteogenesis of hMSCs in Osteoblast Construct in vitro. (A) Total alkaline phosphatase (ALP) activity per scaffold. The ALP activity level was compared between scaffolds with hMSCs, osteoblast-like cells differentiated from hMSCs and standard osteoblasts. All the experiments were conducted independently $(n=3)$. Error bars represent SD. The asterisks $(* *)$ indicate statistically significant differences between different constructs $(P<0.01)$. (B) Osteoblast marker gene expression of cells in PLGA scaffold. Lane1: Osteoblast Construct. Lane 2: Scaffold with standard osteoblasts as a positive control. Lane 3: hMSC Construct as a negative control.

medium for preparation of Osteoblast Construct, the final cell density reached $(2.33 \pm 0.31) \times 10^{6}$ cells $/$ scaffold, which indicated a small increase of cell number after culture with the osteogenic medium and no significant difference in cell density between Osteoblast Construct and hMSC Construct $(P>0.05)$ (Fig. 1B).

\section{Osteogenic differentiation of hMSCs in PLGA scaffolds}

The alkaline phosphatase (ALP) activity assay was used to determine the activity of protein specific for osteogenesis. The PLGA scaffolds with the same density of standard osteoblasts (HCO4600, Yuhengfeng, Beijing, China) and hMSCs were used as positive and negative controls, respectively. The results showed that the level of ALP activity in the Osteoblast Construct (64.5 $\pm 7.4 \mu \mathrm{mol} /$ scaffold) was higher than that in the PLGA scaffold with
hMSCs $(10.7 \pm 2.7 \mu \mathrm{mol} / \mathrm{scaffold})(P<0.01)$ (Fig. 2A). There was no significant difference in ALP activity between the Osteoblast Construct and the PLGA scaffold with standard osteoblasts $(72.3 \pm 9.9 \mu \mathrm{mol} / \mathrm{scaffold})(P>$ $0.05)$. RT-PCR was used to detect the gene expression of osteogenic markers. As shown in Fig. 2B, ALP, COL I and OPN were expressed in the Osteoblast Construct similar to the PLGA scaffold with the standard osteoblasts, while the PLGA scaffold with undifferentiated hMSCs showed a negative result. Therefore, the above results showed that hMSCs in PLGA scaffolds could be induced into osteoblast-like cells after perfusion culture for 14 days with the osteogenic medium.

\section{Macroscopic view post-surgery}

The animals were sacrificed respectively at 10 and 20 weeks after transplantation and the whole calvaria were 

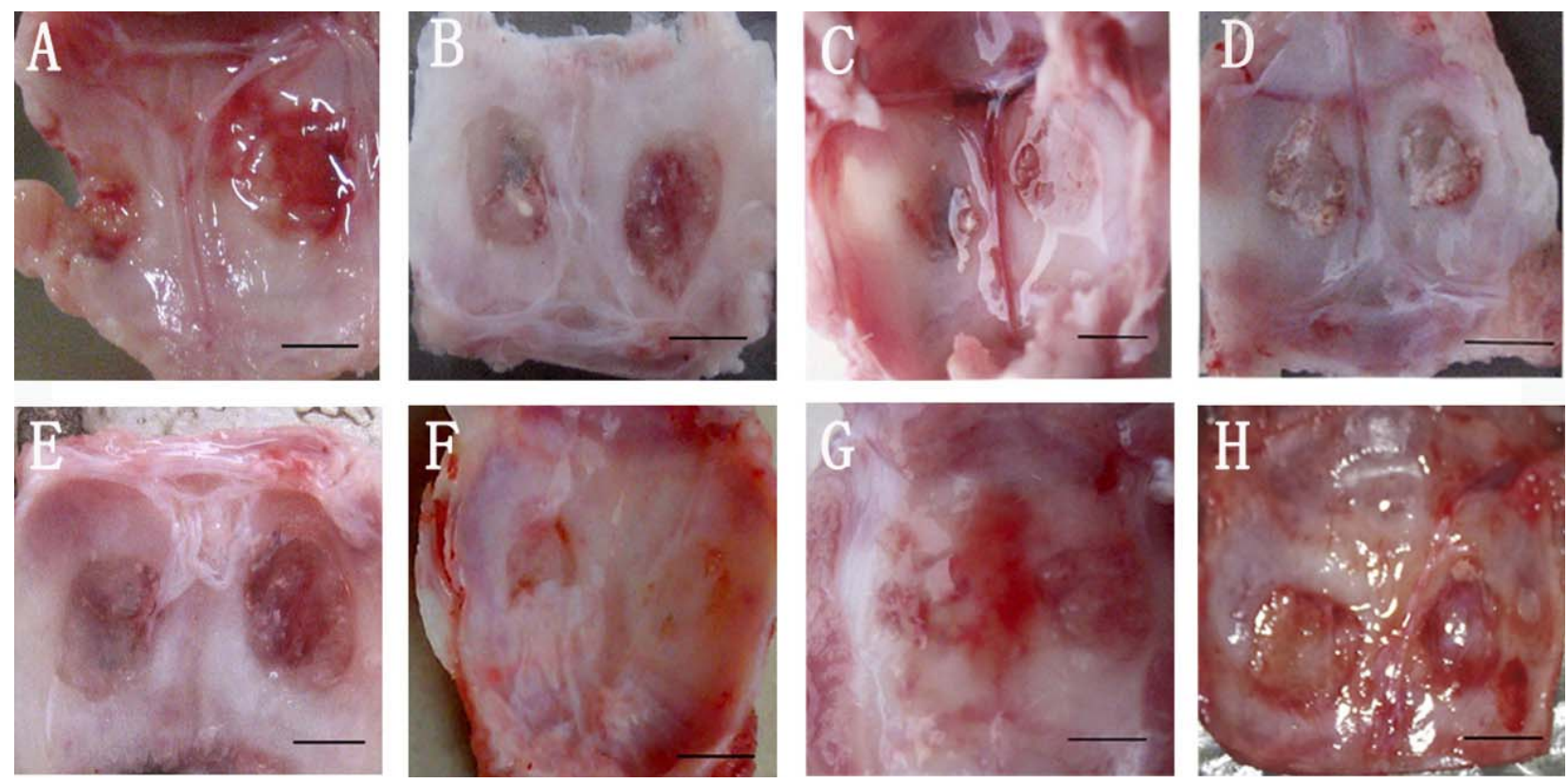

Fig. 3. Macroscopic view of rat calvarias after transplantation. (A)-(D) The graft areas of calvaria at 10 weeks after transplantation. (E)-(H) The graft area of calvaria at 20 weeks after transplantation. The left defect of calvaria in (A) and (E) was grafted with Blank Defect while the right defect was grafted with the hMSC Construct. The left defect of calvaria in (B) and (F) was grafted with Blank Scaffold while the right defect was grafted with the hMSC Construct. The left defect of calvaria in (C) and (G) was grafted with Blank Scaffold and the right defect was grafted with the Osteoblast Construct. The left defect of calvaria in (D) and (H) was grafted with the hMSC Construct and the right defect was grafted with the Osteoblast Construct. Scale bar: $5 \mathrm{~mm}$.
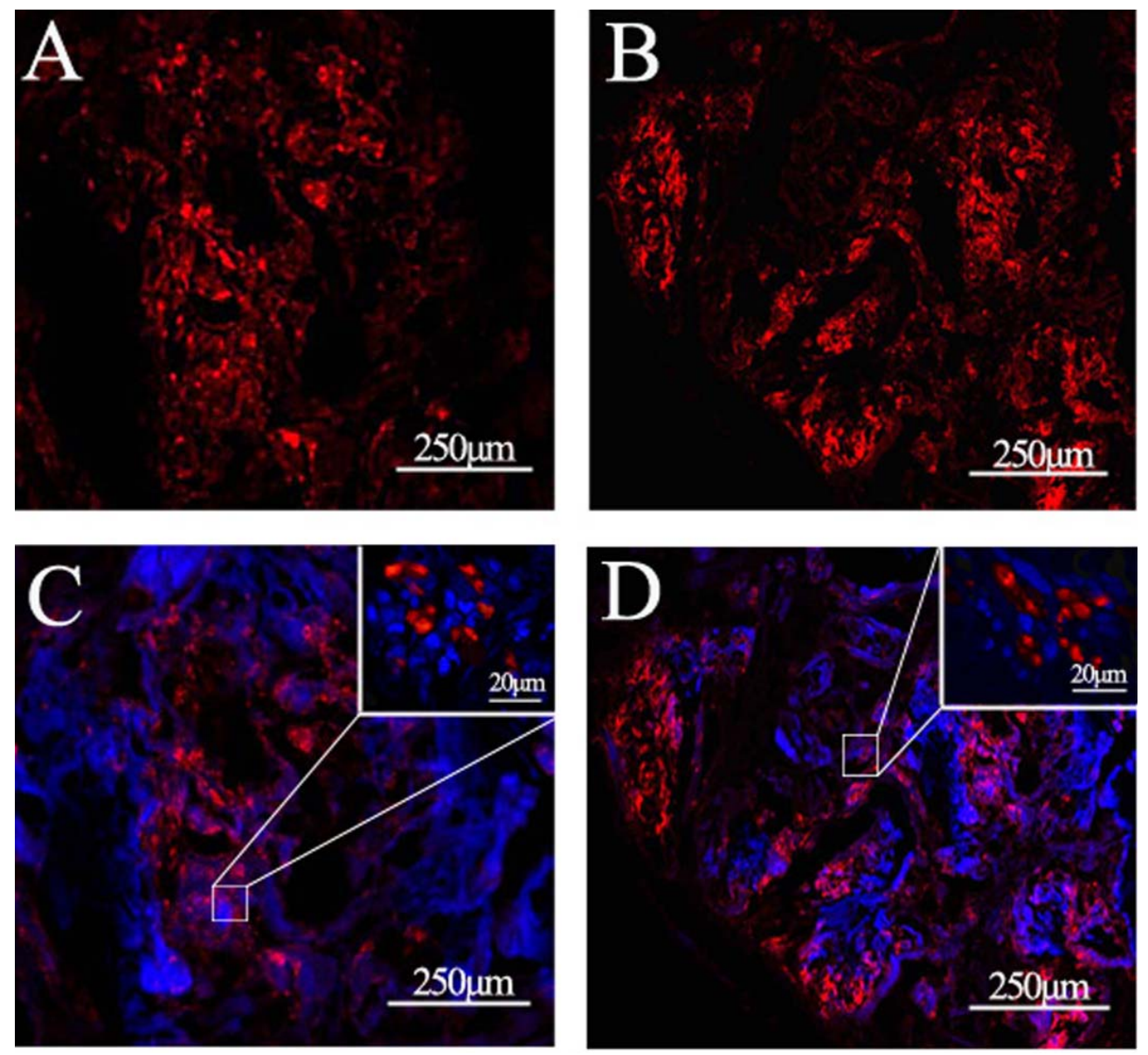

Fig. 4. Cell tracing in vivo at 10 weeks after transplantation. Cells in PLGA scaffold were labeled by fluorescent carbocyanine CM-Dil. (A) and (B) are staining results of hMSCs and osteoblast-like cells differentiated from hMSCs respectively. The red staining represents human cells survival in rats after transplantation. (C) and (D) are counterstaining results with DAPI. The blue staining show native cells in rats. 
Blank defect
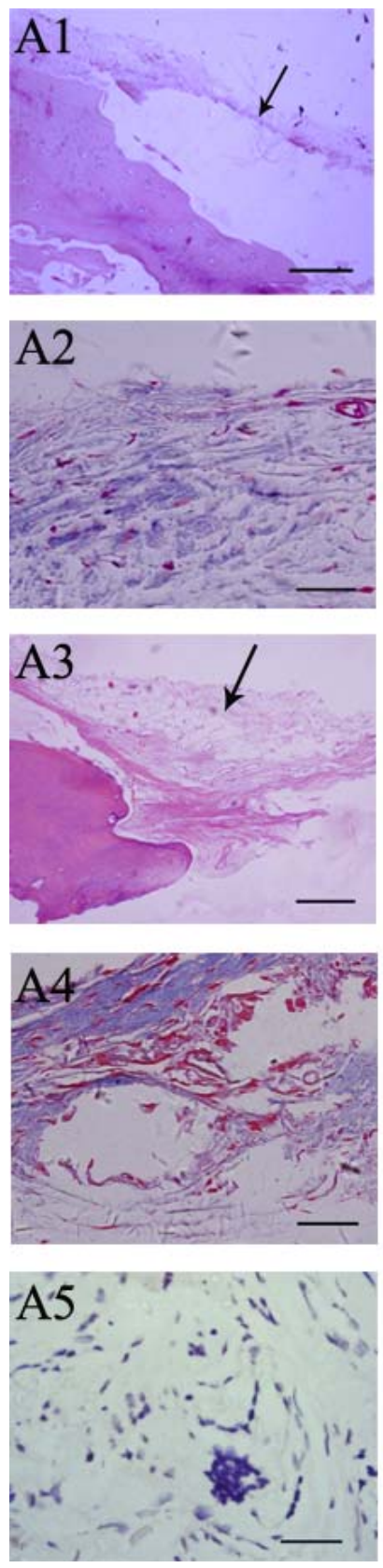

Fig. 5. Histological and immunohistochemical analysis of graft areas after transplantation. Defect areas were treated with Blank Defect (A), Blank Scaffold (B), hMSCs Construct (C) and Osteoblast Construct (D). 1 series: General images (A1-D1) at 10 weeks after transplantation, 2 series: Masson's Trichrome Staining images (A2-D2) showing the vascularization in the graft area at 10 weeks after transplantation. 3 series: General images (A3-D3) showing the bone formation at 20 weeks after transplantation. 4 series: Images (A4-D4) showing collagen produced in the newly formed tissues by Masson's Trichrome Staining at 20 weeks after transplantation. 5 series: Images (A5-D5) showing human osteocalcin (OCN) in the graft areas by immunohistochemistry assay at 20 weeks after transplantation. HB: host bone. NB: new bone.
hMSC Construct
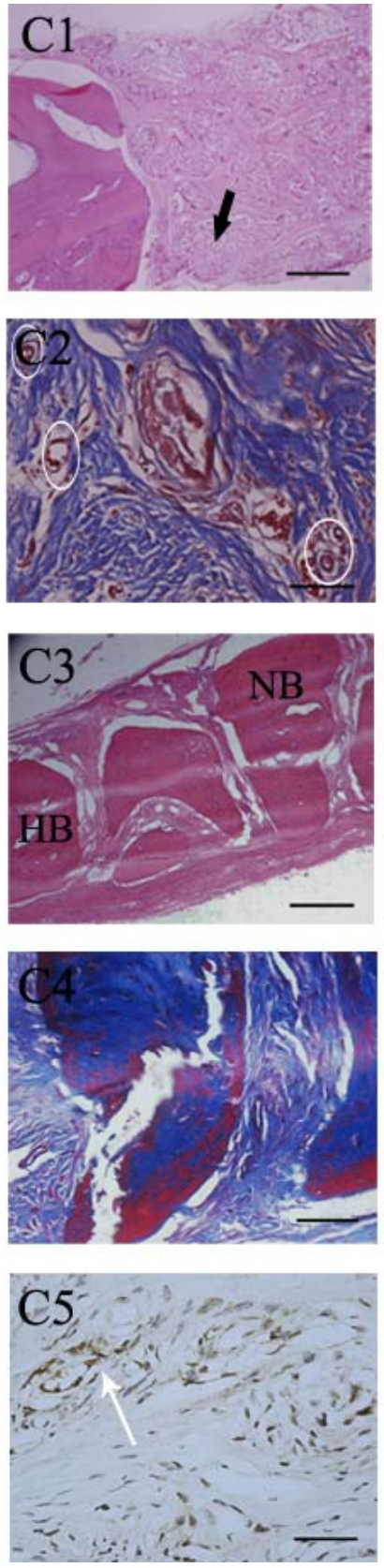

$\mathbf{E}$

\section{Osteoblast Construct}
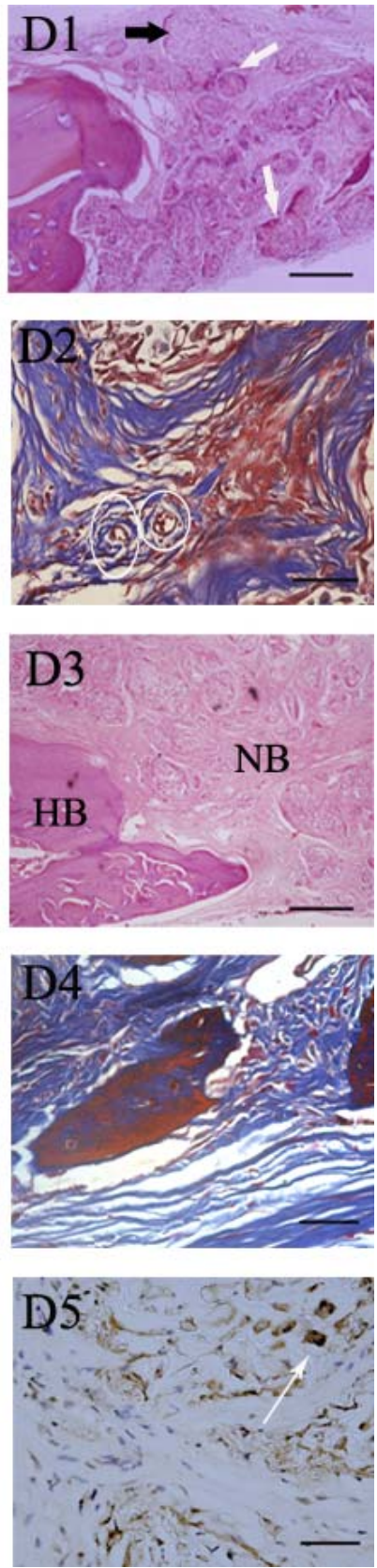

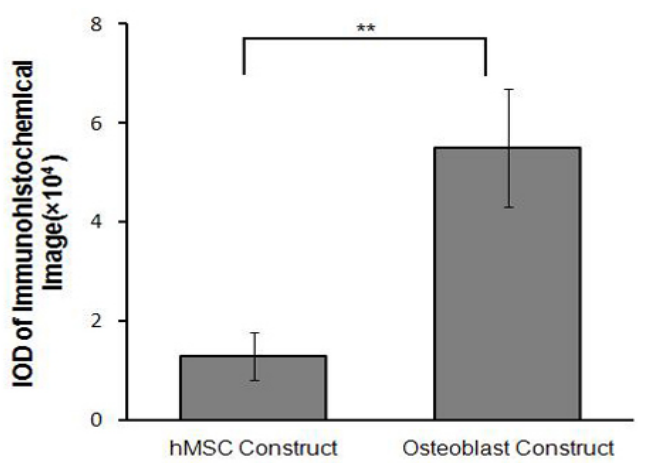

The thin black arrow: the formatted membrane. The thick black arrow: the residual scaffold material. The thin white arrow: the positive staining with human OCN. The thick white arrow: the inflammatory cells. The white oval: the area

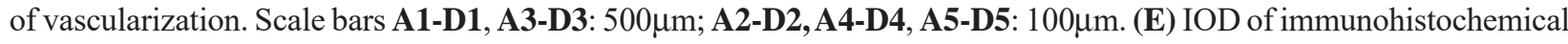
image against human OCN in the area grafted with hMSC Construct and Osteoblast Construct. IOD of the images represent the protein content in the area. The error bar represents SD. The asterisks $(* *)$ indicate a statistically significant difference of IOD between the graft area with hMSC Construct and the graft area with Osteoblast Construct $(P<0.01$, $\mathrm{n}=8)$. 
harvested for macroscopic examination. Both at 10 weeks and at 20 weeks after transplantation, we found only a thin membrane in the defect area without any graft (Blank Defect; Fig. 3A and E). However, fibrous-like connective tissue was observed in the defect area grafted with Blank Scaffold, hMSC Construct or Osteoblast Construct at 10 weeks after transplantation. In the graft areas, most of the scaffold materials were degraded and absorbed. Only a few small scaffold grains remained in the graft area (Fig. 3B-D). In a comparison of three grafts, the fibrous-like connective tissue filled the whole area grafted with hMSC Construct or Osteoblast Construct. However, the fibrouslike connective tissue was observed only at the edge of the area grafted with Blank Scaffold. A thin membrane remained in the central region of the graft area. No significant difference in the morphology of the fibrouslike tissue was observed between the areas grafted with hMSC Construct and Osteoblast Construct. At 20 weeks after transplantation, scaffold materials were no longer observed in any of the graft areas (Fig. 3F-H). Bone-like tissue filled in the areas grafted with both the hMSC Construct and the Osteoblast Construct. However, the bone-like tissue in the area grafted with hMSC Construct $(1.23 \pm 0.12 \mathrm{~mm})$ was thicker than the tissue in the area grafted with Osteoblast Construct $(0.99 \pm 0.14 \mathrm{~mm})(P<$ $0.05)$. In comparison with the areas grafted with hMSC Construct and Osteoblast Construct, the area grafted with Blank Scaffold was still filled with a fibrous-like tissue. These results showed that both hMSC Construct and Osteoblast Construct had the ability to enhance the reconstruction of calvarial defects of rats.

\section{Cell tracing in vivo}

At 10 weeks after transplantation, the graft area was harvested for cell tracing analysis. Under fluorescein microscopy, we detected numerous labeled hMSCs and hMSCs-derived osteoblast-like cells in the graft area. By visual inspection, these cells seemed to have an inhomogeneous distribution in the graft area, and the distribution of osteoblast-like cells seemed to be a little more abundant than that of hMSCs (Fig. 4A and B). Counterstaining with DAPI showed that the space without human cells was filled with native cells of rat (blue staining) (Fig. 4C and D), and the distribution of native cells in the area grafted with hMSC Construct seemed to be more abundant than that in the area grafted with Osteoblast Construct.

\section{Histological analysis}

The reconstruction process of bone in the calvarial defect was evaluated by histological analysis. Representative histological images are showed in Fig. 5. After 10 weeks of transplantation, the defect area without any graft (Blank Defect) was quite different from other graft areas, being lack of any bone ingrowth. Fig. 5A1 showed only a thin membrane in the defect area without any graft. However, the connective tissue was produced in the area grafted with Blank Scaffold, hMSC Construct or Osteoblast Construct. The scaffolds had been greatly degraded except for some residual scaffold materials scattered in the graft area.

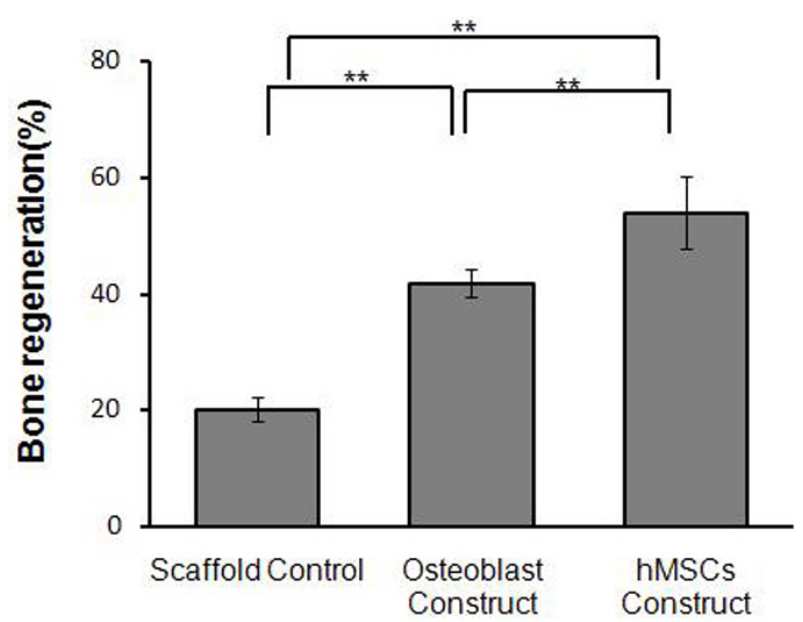

Fig. 6. Bone regeneration percentage of the defect area at 20 weeks after transplantation. The three bars represent the different bone regeneration ability of Blank Scaffold, hMSC Construct and Osteoblast Construct. A statistically significant difference was shown $(P<0.01$, $\mathrm{n}=8$ ) between hMSC Construct and Osteoblast Construct or between hMSC Construct and Blank Scaffold $(P<0.01, \mathrm{n}=8)$.

However, there was still a difference in scaffold degradation between areas grafted with these three grafts. Scaffold materials remained more in the area grafted with Blank Scaffold than in the area grafted with hMSC Construct or Osteoblast Construct. In addition, Fig. 5B1D1 reflect the difference in inflammatory reaction in the graft areas. In comparison to areas grafted with Blank Scaffold, hMSC Construct and Osteoblast Construct, no uninucleate macrophage and multinucleated inflammatory cells were found in the area grafted with Blank Scaffold and hMSC Construct, but the area grafted with Osteoblast Construct showed some inflammatory cells around the scaffold material. In addition, some vessel-like tissue was detected in the areas grafted with hMSC Construct, Osteoblast Construct and Blank Scaffold by Masson's Trichome staining (Fig. 5B2-D2), and the area grafted with hMSC Construct appeared to show a superior vessel-like structuralization.

At 20 weeks after transplantation, there was no obvious change in the defect area without any graft (Fig. 5A3) in comparison with that at 10 weeks after transplantation. No classical bone formation was observed in the area grafted with Blank Scaffold, and some scaffold materials still remained in the graft area (Fig. 5B3). However, no residual scaffold material was detected in both areas grafted with hMSC Construct and Osteoblast Construct (Fig. 5C3D3). The obvious small lump bone was formed in these graft areas, especially in the area grafted with hMSC Construct. In comparison with two areas grafted with hMSC Construct and Osteoblast Construct, the area grafted with hMSC Construct showed some larger woven bone. In addition, collagen, a major extracellular matrix, was produced in the newly formed tissues, as determined by Masson's Trichrome Staining (Fig. 5B4-D4). The bone reconstruction percentage analysis was performed by 
Image-Pro Plus. Fig. 6 indicated that the new bone formation rate in the areas grafted with hMSC Construct $(53.9 \pm 6.2 \%)$ and Osteoblast Construct $(41.8 \pm 2.5 \%)$ was higher than that in the area grafted with Blank Scaffold $(20.1 \pm 2.1 \%)(P<0.01)$. In addition, there was also a significant difference in new bone formation percentage between the area grafted with hMSCs Construct and the area grafted with Osteoblast Construct $(P<0.01)$.

The immunohistochemical analysis specific to human cells was performed to evaluate the survival and function of hMSC and hMSC-derived osteoblast-like cells in the xenogenic transplantation. The area without any graft or with Blank Scaffold did not show the positive staining of human osteocalcin (Fig. 5A5 and 5B5). The areas grafted with hMSC Construct and Osteoblast Construct showed the positive staining of human osteocalcin (Fig. 5C5 and 5D5). However, in comparison of staining images for human osteocalcin between Fig. 5C5 and Fig. 5D5, the expression level of human osteocalcin in the area grafted with Osteoblast Construct was higher than that in the area grafted with hMSC Construct (Fig. 5E, $P<0.01$ ). The negative control treated only with the second antibody showed no any positive staining (no data shown).

\section{Discussion}

Autologous transplantation is an optimal choice of bone repairing treatment. However, xenogenic transplantation may also have a practical significance in future clinical applications, especially in the bone repairing treatment for aged patients. Aged patients with tissue defects may need an allograft transplantation surgery typically because the proliferation and differentiation capacities of stem cells decline in an age-dependent way and may even nearly disappear (Hermann et al., 2010). Therefore, xenogenic transplantation may be an optimal choice to perform tissue reconstruction for those aged recipients in the future. Niemeyer et al. (Niemeyer et al., 2010b) reported the transplantation of human mesenchymal stem cells in a nonautogenous setting for bone regeneration in a rabbit criticalsize defect model. In the present study, the xenogenic transplantation experiment was performed to determine the effect of hMSCs and osteoblast-like cells differentiated from hMSCs on the reconstruction of calvarial defects in rats, especially to compare the bone reconstruction ability of undifferentiated hMSCs with that of osteoblast-like cells differentiated from hMSCs in the xenogenic transplantation model. As a prerequisite for comparison of bone reconstruction abilities between hMSC Construct and Osteoblast Construct, the initial cell densities in these two constructs should be equal. During induction of hMSCs in PLGA scaffolds into osteoblast-like cells, we found that cells still increased to a small extent. Therefore, to decrease the difference in cell densities between hMSC Construct and Osteoblast Construct as much as possible, hMSC Construct was prepared by undergoing 12-dayculture in the growth medium, whereas Osteoblast Construct was prepared as that the PLGA scaffold with hMSCs undergoing 9-day-culture in the growth medium was continued in the osteogenic medium for another 14 days. The cell number increased during 14-day-induction which could compensate for the difference in cell densities between scaffolds for 9-day-cultures and 12-day-cultures in the growth medium.

Critical size defect (CSD) experimental models are essential for in vivo experiments for bone reconstruction. The calvarial CSD of the rat is one of the models for new bone reconstruction and especially a good model for flat bone tissue engineering because the calvaria are suitable for the creation of defects, implantation of grafts, and analysis of reconstruction (Hollinger and Kleinschmidt, 1990; Sweeney et al., 1995). In previous studies, the small defect in $3 \mathrm{~mm}$ diameter could undergo spontaneous bone regeneration. However, the defect in $5 \mathrm{~mm}$ diameter is beyond the size of spontaneous bone regeneration (Aybar Odstrcil et al., 2005; Wurzler et al., 1998). Therefore, the $5 \mathrm{~mm}$ diameter of bilateral full-thickness calvarial defects in 2-month-old SD rats, a proper size of CSD proved by Bosch (Bosch et al., 1998), were used in the present transplantation experiment. In the present experiment, no significant bone formation except for a thin membrane was observed in the defect area without any graft. This demonstrated that the $5 \mathrm{~mm}$ diameter defect is beyond the size of spontaneous bone reconstruction.

Some studies have shown that PLGA could support MSC proliferation and differentiation both in vitro and in vivo (Ishaug-Riley et al., 1997; Karp et al., 2003), and the porous PLGA scaffolds have been utilized for the reconstruction of bone including mandible and diaphysis (Ren et al., 2005; Yoon et al., 2007). We also used the PLGA scaffold without any cells as a control to assess the promotion effect of hMSCs or osteoblast-like cells differentiated from hMSCs on the bone reconstruction of rat calvaria. The PLGA scaffold used in the present experiment was fabricated without any xenogenic growth factors so as to reflect really the bone reconstruction ability of hMSCs or osteoblast-like cell differentiated from hMSCs. As in the previous report (Ren et al., 2005), the PLGA scaffold alone could not reconstruct the whole larger defect area except for formation of bone-like tissue at the rim of defect. The porous scaffold is only a carrier for cell creeping and has no bone inducement function. The key aim of our experiment was to compare the bone reconstruction capacities between undifferentiated hMSCs and osteoblast-like cells differentiated from hMSCs. Significant bone formation was observed in the area grafted with hMSC Construct or Osteoblast Construct. The thickness of new bone formed in the graft area was thinner than that of rat calvaria surrounding the graft area, but the new bone in the graft area was relatively compact. As in the macroscopic view and histological assay described above, the undifferentiated hMSCs seemed to have a superior ability of bone reconstruction in comparison with that of osteoblast-like cells differentiated from hMSCs. According to the histological assay, the new bone formation in the area grafted with hMSC Construct was superior to that in the area grafted with Osteoblast Construct. This was confirmed by the statistical analysis of bone formation. Of course, micro CT is a standard tool 
to quantify bone regeneration. Regretfully, there was no the micro CT instrument available to measure quantitatively the new bone formation. Some other methods such as macroscopic examination, histological assay and semi-quantitative bone formation analysis, however, may be acceptable for evaluation of bone reconstruction in rat calvaria.

Our result that undifferentiated hMSCs induced more new bone formation in the graft area seems in contradiction to a previous study on the athymic mice transplantation model (Meinel et al., 2005). According to the analysis of cell tracing in vivo, both hMSC and osteoblast-like cells differentiated from hMSCs survived in rats. However, the abundance of hMSCs seemed to be less than that of osteoblast-like cells. It should be inferred that the effect of the hMSCs on the promotion of bone reconstruction of xenogenic animals lies not only on the osteogenic differentiation of hMSCs to reconstruct the defect directly (Kim et al., 2007), but also on their indirect function in the xenogenic transplantation, e.g., hMSCs may secret some growth factors to recruit native cells into the defect area to generate new osseous tissues (Bi et al., 1999; Frank et al., 2002). As shown in Fig. 4, there were native cells (in blue) surrounding the transplanted cells (in red). It is supposed that these native cells are translocated from the native environment surrounding the graft area. In addition, the distribution of native cells in the defect area grafted with hMSC Construct appeared to be more abundant in comparison with that in the defect area grafted with Osteoblast Construct. If this difference had been verified by further studies, we could infer that the hMSCs should have superior ability of recruiting native cells compared to osteoblast-like cells. Of course, it is also possible that the signal shown in Fig. 4 is from macrophages digesting labeled hMSCs or rat cells labeled due to in vivo label transfer. This possibility should be explored in further studies.

The above inference should also be confirmed by our immunohistochemistry assay of human osteocalcin. Although the positive staining rate of osteocalcin from human in the area grafted with Osteoblast Construct was higher than that in the area grafted with hMSC Construct, the total bone reconstruction in the area grafted with hMSC Construct was superior to that in the area grafted with Osteoblast Construct. In addition, stem cells may feel signals from the microenvironment and thus differentiate into vascular endothelial cells, or send out signals to recruit endothelial progenitor cells from the circulating blood. These effects are contributed to angiogenesis that is one of the most important factors for bone formation (Kim et al., 2007). However, osteoblast-like cells differentiated from hMSCs may lose the ability to differentiate into endothelial cells and hence lose the potential for angiogenesis. As shown in Fig. 5C2 and D2, a vessel-like structure in the area grafted with hMSCs Construct and Osteoblast Construct was detected, and the formation of the vessel-like structure in the area grafted with hMSC Construct was superior to that in the area grafted with Osteoblast Construct. However, it should be verified by the vascular specific staining in a further study whether the formation of a vessel-like structure in the graft area is indeed a sign of angiogenesis.

The immunogenicity of allogeneic cells in the recipient is an important problem in clinical cell transplantation. Previous studies demonstrated that both undifferentiated and differentiated MSCs have low immunogenic profiles (Le Blanc et al., 2003a; Le Blanc et al., 2003b; Pierdomenico et al., 2005; Niemeyer et al., 2007). Those studies showed that MSCs do not elicit alloreactive lymphocyte responses in vitro due to their special immune characteristics. Nevertheless, Poncelet et al. (Poncelet et al., 2007) found that xenogenic MSCs with low immunogenicity in vitro elicited an immune response in vivo after transplantation. In our experiments, an inflammatory reaction, such as production of macrophages, was not observed in the area grafted with hMSC Construct. However, this reaction was elicited in the area grafted with Osteoblast Construct, especially in the initial period after transplantation. Though this is not enough to prove the presentation of a local immune response, it can be an indication that a local inflammation occurred. The effect of the PLGA scaffold on the inflammatory reaction was excluded since the same reaction was not elicited in the area grafted with Blank Scaffold. Therefore, it is deduced that the immunogenic characteristic of osteoblast-like cells differentiated from hMSCs should be different from that of hMSCs or that the immunogenic characteristics of osteoblast-like cells should have changed in vivo after transplantation. The difference of immunogenic characteristics in vitro and in vivo between hMSCs and osteoblast-like cells differentiated from hMSCs should be a subject for further research.

\section{Conclusion}

In conclusion, the present study demonstrates that hMSC enable bone reconstruction of calvarial defects in a xenogenic transplantation model and may have superior potential of bone reconstruction compared to osteoblastlike cells according to morphological observations and histochemical analysis.

\section{Acknowledgements}

We would like to thank three anonymous reviewers for critical comments on this manuscript. This study was financially supported by Scientific Research from Scientific Fund of Zhejiang (2009C13020) and National Natural Science Fund of China (30971460).

\section{References}

Ahmad S, Ahmed A (2004) Elevated placental soluble vascular endothelial growth factor receptor-1 inhibits angiogenesis in preeclampsia. Circ Res 95: 884-891.

Aybar Odstrcil A, Territoriale E, Missana L (2005) An experimental model in calvaria to evaluate bone therapies. Acta Odontol Latinoam 18: 63-67.

Bernardo ME, Avanzini MA, Perotti C, Cometa AM, Moretta A, Lenta E, Del Fante C, Novara F, de Silvestri A, 
Amendola G, Zuffardi O, Maccario R, Locatelli F (2007) Optimization of in vitro expansion of human multipotent mesenchymal stromal cells for cell-therapy approaches: further insights in the search for a fetal calf serum substitute. J Cell Physiol 211: 121-130.

Bi LX, Simmons DJ, Mainous E (1999) Expression of BMP-2 by rat bone marrow stromal cells in culture. Calcif Tissue Int 64: 63-68.

Bosch C, Melsen B, Vargervik K (1998) Importance of the critical-size bone defect in testing bone-regenerating materials. J Craniofac Surg 9: 310-316.

Bruder SP, Kraus KH, Goldberg VM, Kadiyala S (1998a) The effect of implants loaded with autologous mesenchymal stem cells on the healing of canine segmental bone defects. J Bone Joint Surg Am 80: 985-996.

Bruder SP, Kurth AA, Shea M, Hayes WC, Jaiswal N, Kadiyala S (1998b) Bone regeneration by implantation of purified, culture-expanded human mesenchymal stem cells. J Orthop Res 16: 155-162.

Cowan CM, Shi YY, Aalami OO, Chou YF, Mari C, Thomas R, Quarto N, Contag CH, Wu B, Longaker MT (2004) Adipose-derived adult stromal cells heal criticalsize mouse calvarial defects. Nat Biotechnol 22: 560-567.

Cui L, Liu B, Liu G, Zhang W, Cen L, Sun J, Yin S, Liu W, Cao Y (2007) Repair of cranial bone defects with adipose derived stem cells and coral scaffold in a canine model. Biomaterials 28: 5477-5486.

Cybernetics M (2002) Image-Pro Plus - application notes. Media Cybernetics, Silver Spring, MD.

Ferrari A, Hannouche D, Oudina K, Bourguignon M, Meunier A, Sedel L, Petite H (2001) In vivo tracking of bone marrow fibroblasts with fluorescent carbocyanine dye. J Biomed Mater Res 56: 361-367.

Frank O, Heim M, Jakob M, Barbero A, Schafer D, Bendik I, Dick W, Heberer M, Martin I (2002) Real-time quantitative RT-PCR analysis of human bone marrow stromal cells during osteogenic differentiation in vitro. $\mathrm{J}$ Cell Biochem 85: 737-746.

Garvey W (1984) Modified elastic tissue - Masson trichrome stain. Stain Technol 59: 213-216.

Gong Z, Niklason LE (2008) Small-diameter human vessel wall engineered from bone marrow-derived mesenchymal stem cells (hMSCs). FASEB J 22: $1635-$ 1648.

Gopferich A (1996) Mechanisms of polymer degradation and erosion. Biomaterials 17: 103-114.

Hao W, Pang L, Jiang M, Lv R, Xiong Z, Hu YY (2010) Skeletal repair in rabbits using a novel biomimetic composite based on adipose-derived stem cells encapsulated in collagen I gel with PLGA-beta-TCP scaffold. J Orthop Res 28: 252-257.

Hermann A, List C, Habisch HJ, Vukicevic V, EhrhartBornstein M, Brenner R, Bernstein P, Fickert S, Storch A (2010) Age-dependent neuroectodermal differentiation capacity of human mesenchymal stromal cells: limitations for autologous cell replacement strategies. Cytotherapy 12: 17-30.

Hollinger JO, Kleinschmidt JC (1990) The critical size defect as an experimental model to test bone repair materials. J Craniofac Surg 1: 60-68.
Holtorf HL, Jansen JA, Mikos AG (2006) Modulation of cell differentiation in bone tissue engineering constructs cultured in a bioreactor. Adv Exp Med Biol 585: 225-241.

Ishaug-Riley SL, Crane GM, Gurlek A, Miller MJ, Yasko AW, Yaszemski MJ, Mikos AG (1997) Ectopic bone formation by marrow stromal osteoblast transplantation using poly(DL-lactic-co-glycolic acid) foams implanted into the rat mesentery. J Biomed Mater Res 36: 1-8.

Jorgensen C, Gordeladze J, Noel D (2004) Tissue engineering through autologous mesenchymal stem cells. Curr Opin Biotechnol 15: 406-410.

Karp JM, Shoichet MS, Davies JE (2003) Bone formation on two-dimensional poly(DL-lactide-coglycolide) (PLGA) films and three-dimensional PLGA tissue engineering scaffolds in vitro. J Biomed Mater Res A 64: 388-396.

Kassem M, Risteli L, Mosekilde L, Melsen F, Eriksen EF (1991) Formation of osteoblast-like cells from human mononuclear bone marrow cultures. APMIS 99: 269-274.

Kim J, Kim IS, Cho TH, Lee KB, Hwang SJ, Tae G, Noh I, Lee SH, Park Y, Sun K (2007) Bone regeneration using hyaluronic acid-based hydrogel with bone morphogenic protein-2 and human mesenchymal stem cells. Biomaterials 28: 1830-1837.

Koc ON, Gerson SL, Cooper BW, Dyhouse SM, Haynesworth SE, Caplan AI, Lazarus HM (2000) Rapid hematopoietic recovery after coinfusion of autologousblood stem cells and culture-expanded marrow mesenchymal stem cells in advanced breast cancer patients receiving high-dose chemotherapy. J Clin Oncol 18: 307316.

Kon E, Muraglia A, Corsi A, Bianco P, Marcacci M, Martin I, Boyde A, Ruspantini I, Chistolini P, Rocca M, Giardino R, Cancedda R, Quarto R (2000) Autologous bone marrow stromal cells loaded onto porous hydroxyapatite ceramic accelerate bone repair in criticalsize defects of sheep long bones. J Biomed Mater Res 49: 328-337.

Korda M, Blunn G, Goodship A, Hua J (2008) Use of mesenchymal stem cells to enhance bone formation around revision hip replacements. J Orthop Res 26: 880-885.

Kubler N, Michel C, Zoller J, Bill J, Muhling J, Reuther J (1995) Repair of human skull defects using osteoinductive bone alloimplants. J Craniomaxillofac Surg 23: 337-346.

Langer R, Vacanti JP (1993) Tissue engineering. Science 260: 920-926.

Lazarus HM, Koc ON, Devine SM, Curtin P, Maziarz RT, Holland HK, Shpall EJ, McCarthy P, Atkinson K, Cooper BW, Gerson SL, Laughlin MJ, Loberiza FR Jr, Moseley AB, Bacigalupo A (2005) Cotransplantation of HLA-identical sibling culture-expanded mesenchymal stem cells and hematopoietic stem cells in hematologic malignancy patients. Biol Blood Marrow Transplant 11: 389-398.

Le Blanc K, Tammik C, Rosendahl K, Zetterberg E, Ringden O (2003a) HLA expression and immunologic properties of differentiated and undifferentiated mesenchymal stem cells. Exp Hematol 31: 890-896. 
Le Blanc K, Tammik L, Sundberg B, Haynesworth SE, Ringden O (2003b) Mesenchymal stem cells inhibit and stimulate mixed lymphocyte cultures and mitogenic responses independently of the major histocompatibility complex. Scand J Immunol 57: 11-20.

Mankani MH, Kuznetsov SA, Wolfe RM, Marshall GW, Robey PG (2006) In vivo bone formation by human bone marrow stromal cells: reconstruction of the mouse calvarium and mandible. Stem Cells 24: 2140-2149.

Meinel L, Fajardo R, Hofmann S, Langer R, Chen J, Snyder B, Li C, Zichner L, Langer R, Vunjak-Novakovic G, Kaplan DL (2005) Silk implants for the healing of critical size bone defects. Bone 37: 688-698.

Miura M, Miura Y, Sonoyama W, Yamaza T, Gronthos S, Shi S (2006) Bone marrow-derived mesenchymal stem cells for regenerative medicine in craniofacial region. Oral Dis 12: 514-522.

Niemeyer P, Kornacker M, Mehlhorn A, Seckinger A, Vohrer J, Schmal H, Kasten P, Eckstein V, Südkamp NP, Krause U (2007) Comparison of immunological properties of bone marrow stromal cells and adipose tissue-derived stem cells before and after osteogenic differentiation in vitro. Tissue Eng 13: 111-121.

Niemeyer P, Schonberger TS, Hahn J, Kasten P, Fellenberg J, Suedkamp N, Mehlhorn AT, Milz S, Pearce S (2010a) Xenogenic transplantation of human mesenchymal stem cells in a critical size defect of the sheep tibia for bone regeneration. Tissue Eng Part A 16: 33-43.

Niemeyer P, Szalay K, Luginbuhl R, Südkamp NP, Kasten P (2010b) Transplantation of human mesenchymal stem cells in a non-autogenous setting for bone regeneration in a rabbit critical-size defect model. Acta Biomater 6: 900-908.

Pierdomenico L, Bonsi L, Calvitti M, Rondelli D, Arpinati M, Chirumbolo G, Becchetti E, Marchionni C, Alviano F, Fossati V, Staffolani N, Franchina M, Grossi A, Bagnara GP (2005) Multipotent mesenchymal stem cells with immunosuppressive activity can be easily isolated from dental pulp. Transplantation 80: 836-842.

Poncelet AJ, Vercruysse J, Saliez A, Gianello P (2007) Although pig allogeneic mesenchymal stem cells are not immunogenic in vitro, intracardiac injection elicits an immune response in vivo. Transplantation 83: 783-790.
Ren T, Ren J, Jia X, Pan K (2005) The bone formation in vitro and mandibular defect repair using PLGA porous scaffolds. J Biomed Mater Res A 74: 562-569.

Sweeney TM, Opperman LA, Persing JA, Ogle RC (1995) Repair of critical size rat calvarial defects using extracellular matrix protein gels. J Neurosurg 83: 710-715.

Wurzler KK, DeWeese TL, Sebald W, Reddi AH (1998) Radiation-induced impairment of bone healing can be overcome by recombinant human bone morphogenetic protein-2. J Craniofac Surg 9: 131-137.

Xie CG, Wang JF, Xiang Y, Jia BB, Qiu LY, Wang LJ, Wang GZ, Huang GP (2005) Marrow mesenchymal stem cells transduced with TPO/FL genes as support for ex vivo expansion of hematopoietic stem/progenitor cells. Cell Mol Life Sci 62: 2495-2507.

Yamada Y, Ueda M, Naiki T, Takahashi M, Hata K, Nagasaka T (2004) Autogenous injectable bone for regeneration with mesenchymal stem cells and plateletrich plasma: tissue-engineered bone regeneration. Tissue Eng 10: 955-964.

Yang J, Cao C, Wang W, Tong X, Shi D, Wu F, Zheng Q, Guo C, Pan Z, Gao C, Wang J (2010) Proliferation and osteogenesis of immortalized bone marrow-derived mesenchymal stem cells in porous polylactic glycolic acid scaffolds under perfusion culture. J Biomed Mater Res A 93: 817-829.

Yoon SJ, Park KS, Kim MS, Rhee JM, Khang G, Lee HB (2007). Repair of diaphyseal bone defects with calcitriol-loaded PLGA scaffolds and marrow stromal cells. Tissue Eng 13: 1125-1133.

Younger EM, Chapman MW (1989) Morbidity at bone graft donor sites. J Orthop Trauma 3: 192-195.

Zhou QL, Gong YH, Gao CY (2005) Microstructure and mechanical properties of poly(L-lactide) scaffolds fabricated by gelatin particle leaching method. J Appl Polymer Sci 98: 1373-1379.

Zsarnovszky A, Le HH, Wang HS, Belcher SM (2005) Ontogeny of rapid estrogen-mediated extracellular signalregulated kinase signaling in the rat cerebellar cortex: potent nongenomic agonist and endocrine disrupting activity of the xenoestrogen bisphenol A. Endocrinology 146: 5388-5396. 